\title{
Corrections to Mean Square Error Matrix Superiority of Empirical Bayes Estimators Under Misspecification
}

\author{
L. WEI* and G. TRENKLER** \\ * Department of Mathematics, University of Science \\ and Technology of China, Hefei, China \\ ** Department of Statistics, Unicersity of Dormund \\ 44221 Dortmund, Germany
}

\section{SUMMARY}

Corrections to "Mean Square Error Matrix Superiority of Empirical Bayes Estimators Under Misspecification", Test 4 (1995), No.1, pp 187-205.

Page 192-193. Formulas (3.3) to (3.6) should be substituted by

$$
\begin{aligned}
I_{11} & =E\left[\left(\tilde{\theta}_{n}-\theta\right)\left(\tilde{\theta}_{n}-\theta\right)^{\prime}\right] \\
& =\frac{1}{(n+1)^{2}}\left\{\sum_{i=1}^{n} E\left(\hat{\beta}_{i}-\theta\right)\left(\hat{\beta}_{i}-\theta\right)^{\prime}+E(\hat{\beta}-\theta)(\hat{\beta}-\theta)^{\prime}\right\} \\
& =\frac{1}{(n+1)^{2}}\left[\sum_{i=1}^{n} Q_{i}+Q_{0}\right] \\
& =\frac{Q_{0}}{n+1},
\end{aligned}
$$

by virtue of $Q_{i}=\operatorname{cov}\left(\hat{\beta}_{i}\right)=\operatorname{cov}(\hat{\beta})=Q_{0}, i=1, \ldots, n$.

By Lemma 3.1 we obtain

$$
\begin{aligned}
Q_{0} & =E[\operatorname{cov}(\hat{\beta} \mid \beta)]+\operatorname{cov}[E(\hat{\beta} \mid \beta)] \\
& =\left(\sigma^{2}+r^{2}\right)\left(X^{\prime} X\right)^{-1}
\end{aligned}
$$

Received April 1996. 
Page 193-202.

(i) Lines 5 and 6 (i.e., the formula (3.22)) of page 199 should be deleted.

(ii) At the end of line 4 on page 201 one should add the formula number (3.29)

(iii) The numbers of formulas (3.7) to (3.21), (3.23) to (3.31), (3.32) to (3.35) and (3.37) to (3.41) occurring in the formulas should be changed into (3.5) to (3.19), (3.20) to (3.28), (3.30) to (3.33), (3.34) to (3.38) respectively.

All formula numbers in the text of section 3 must remain unchanged. 